Supporting Information for

\title{
Catalytic Enantioselective Desymmetrization of meso Cyclic Anhydrides via Iridium-Catalyzed Hydrogenation **
}

Tang-Lin Liu, Wei Li, Huiling Geng, Chun-Jiang Wang, * Xumu Zhang*

[*]T.-L. Liu, H. Geng, Prof. Dr. C.-J. Wang and Prof. X. Zhang

College of Chemistry and Molecular Sciences

Wuhan University, Wuhan, Hubei, 430072 P. R. China

E-mail: cjwang@whu.edu.cn

[*]T.-L. Liu, Dr. W. Li and Prof. X. Zhang

Department of Chemistry and Chemical Biology \& Department of Pharmaceutical Chemistry

Rutgers, the State University of New Jersey. Piscataway, NJ 08854, USA

E-mail: xumu@rci.rutgers.edu

[**]This work was supported by National Institutes of Health (GM58832), the Fundamental Research Funds for the Central Universities, academic award for excellent Ph.D. Candidates funded by Ministry of Education of China (5052011203011).

\section{Contents}

1. Experimental Section $\quad$ S2

2. General procedure for the hydrogenation of meso-anhydrides S2

3. ${ }^{1} \mathrm{H}$ and ${ }^{13} \mathrm{C}$ NMR spectra of $\gamma$-lactones S5 


\section{General Remarks.}

General Information Unless otherwise noted, all reagents and solvents were purchased from commercial suppliers and used without further purification. NMR spectra were recorded on 400 $\mathrm{MHz}$ for ${ }^{1} \mathrm{H}$ NMR and $100 \mathrm{MHz}$ for ${ }^{13} \mathrm{C} \mathrm{NMR}$. $\mathrm{CDCl}_{3}$ was the solvent used for the NMR analysis, with tetramethylsilane as the internal standard. Chemical shifts were reported upfield to TMS (0.00 ppm) for ${ }^{1} \mathrm{H}$ NMR and relative to $\mathrm{CDCl}_{3}(77.0 \mathrm{ppm})$ for ${ }^{13} \mathrm{C} \mathrm{NMR}$. Optical rotation was determined using a Perkin Elmer 343 polarimeter. GC analysis was performed with an Agilent

7890A GC System. HPLC analysis was conducted on an Agilent 1200 Series instrument. Column chromatography was performed with silica gel Merck 60 (230-400 mesh).

General procedure of the hydrogenation: A stock solution of $[\operatorname{Ir}(\mathrm{COD}) \mathrm{Cl}]_{2}(\mathrm{COD}=$ cycloocta1,5-diene) and $\mathrm{C}_{3}{ }^{*}$-DTBM-TunePhos in a 1:2.2 molar ratio was stirred in EtOAc at room temperature for $30 \mathrm{~min}$ in a nitrogen-filled glovebox. The catalyst solution $(0.1 \mathrm{~mL}, 0.0001$ mmol) was then transferred by syringe into the vials charged with different substrates $(0.1 \mathrm{mmol})$ in EtOAc $(1 \mathrm{~mL})$. All the vials were placed in a steel autoclave, into which hydrogen gas (80 bar) was introduced. After stirring at 80 or $125{ }^{\circ} \mathrm{C}$ for $20 \mathrm{~h}$, the hydrogen was released, the solution was concentrated, and then the crude reaction mixture was eluted (EtOAc) through a short column of silica gel to remove the metal complex. The purified product mixture was analyzed by chiral GC or chiral HPLC to determine the ee value.<smiles>O=C1OC[C@H]2[C@H]1N(Cc1ccccc1)C(=O)N2Cc1ccccc1</smiles>

(3aR,6aS)-1,3-dibenzyltetrahydro-1H-furo[3,4-d]imidazole-2,4-dione ${ }^{[1]}$ (3)

The title compound was prepared according to the general procedure as described above in $>99 \%$ conversion, 96\% yield. ${ }^{1} \mathrm{H}$ NMR $\left(\mathrm{CDCl}_{3}\right.$, TMS, $\left.400 \mathrm{MHz}\right) \delta$ 7.38-7.24 (m, 10H), $5.04(\mathrm{~d}, J=$ $14.8 \mathrm{~Hz}, 1 \mathrm{H}$ ), 4.62 (d, $J=15.2 \mathrm{~Hz}, 1 \mathrm{H}), 4.62$ (dd, $J=7.2 \mathrm{~Hz}, 14.8 \mathrm{~Hz}, 2 \mathrm{H}), 4.15-4.10$ (m, 3H), 3.92 (d, $J=8.8 \mathrm{~Hz}, 1 \mathrm{H}) ;{ }^{13} \mathrm{C}$ NMR $\left(\mathrm{CDCl}_{3}\right.$, TMS, $\left.100 \mathrm{MHz}\right) \delta 172.79,158.31,136.00,135.89$, 
129.06, 128.84, 128.79, 128.61, 128.26, 128.14, 127.92, 54.45, 52.54, 46.99, 45.30; The product was analyzed by HPLC to determine the enantiomeric excess: 96\% ee (Chiralcel OD-H, ipropanol $/$ hexane $=30 / 70$, flow rate $0.5 \mathrm{~mL} / \mathrm{min}, \lambda=220 \mathrm{~nm}$ ); $\mathrm{t}_{\mathrm{r}}=25.85$ and $36.15 \mathrm{~min}$.<smiles>O=C1OCC2CCCCC12</smiles>

\section{(3aS,7aR)-hexahydroisobenzofuran-1(3H)-one ${ }^{[2]}$ (10)}

The title compound was prepared according to the general procedure as described above in $>99 \%$ conversion, 94\% yield. ${ }^{1} \mathrm{H}$ NMR (CDCl 3 , TMS, $\left.400 \mathrm{MHz}\right) \delta 4.20$ (dd, $J=4.8 \mathrm{~Hz}, 8.8 \mathrm{~Hz}, 1 \mathrm{H}$ ), 3.96 (dd, $J=1.2 \mathrm{~Hz}, 8.8 \mathrm{~Hz}, 1 \mathrm{H}$ ), 2.65-2.64 (m, 1H), 2.48 (m, 1H), 2.11-2.10 (m, 2H), 1.68-1.59 (m, 4H), 1.35-1.21 (m, 4H); ${ }^{13} \mathrm{C}$ NMR (CDCl 3 , TMS, $\left.100 \mathrm{MHz}\right) \delta$ 177.45, 70.71, 38.49, 34.43, 26.19, 22.44, 21.95, 21.51; The product was analyzed by GC to determine the enantiomeric excess: $91 \%$ ee (Beta Dex 225 column, $150{ }^{\circ} \mathrm{C}, 1.0 \mathrm{~mL} / \mathrm{min}$,); $\mathrm{t}_{\mathrm{r}}=12.78$ and $14.60 \mathrm{~min}$.

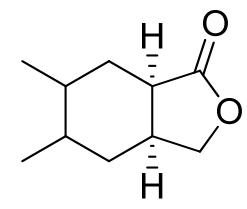

\section{(3aS,7aR)-5,6-dimethylhexahydroisobenzofuran-1(3H)-one (13)}

The title compound was prepared according to the general procedure as described above in $>99 \%$ conversion, 93\% yield. ${ }^{1} \mathrm{H}$ NMR ( $\mathrm{CDCl}_{3}$, TMS, $\left.400 \mathrm{MHz}\right) \delta 4.31$ (dd, $J=5.6 \mathrm{~Hz}, 8.8 \mathrm{~Hz}, 1 \mathrm{H}$ ), 3.97 (dd, $J=2.4 \mathrm{~Hz}, 8.8$ Hz, 1H), 2.77-2.74 (m, 1H), 2.69-2.63 (m, 1H), 2.38-1.85 (m, 4H), 1.66 (d, $J=8.0 \mathrm{~Hz}, 6 \mathrm{H}) ;{ }^{13} \mathrm{C}$ NMR (CDCl 3 , TMS, $\left.100 \mathrm{MHz}\right) \delta$ 179.67, 70.96, 41.77, 36.24, 27.09, 25.67, 24.85, 24.56, 21.60, 19.74; The product was analyzed by GC to determine the enantiomeric excess: 94\% ee (Gama Dex 120 column, $140{ }^{\circ} \mathrm{C}, 1.0 \mathrm{~mL} / \mathrm{min}$ ); $\mathrm{t}_{\mathrm{r}}=39.94$ and 40.23 $\min$.

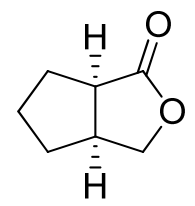


(3aS,6aR)-hexahydro-1H-cyclopenta[c]furan-1-one ${ }^{[3]}$ (15)

The title compound was prepared according to the general procedure as described above in $>99 \%$ conversion, 97\% yield. ${ }^{1} \mathrm{H}$ NMR $\left(\mathrm{CDCl}_{3}\right.$, TMS, $\left.400 \mathrm{MHz}\right) \delta 4.46$ (dd, $\left.J=8.0 \mathrm{~Hz}, 9.2 \mathrm{~Hz}, 1 \mathrm{H}\right)$, 3.99 (dd, $J=2.4 \mathrm{~Hz}, 9.2 \mathrm{~Hz}, 1 \mathrm{H}), 3.01-2.95$ (m, 2H), 2.08-1.43 (m, 6H); ${ }^{13} \mathrm{C} \mathrm{NMR} \mathrm{(CDCl}{ }_{3}$, TMS, $100 \mathrm{MHz}) \delta 181.05,73.58,44.49,38.98,33.74,30.68,25.49$; The product was analyzed by GC

to determine the enantiomeric excess: 80\% ee (Beta Dex 225 column, $150{ }^{\circ} \mathrm{C}, 1.0 \mathrm{~mL} / \mathrm{min}$,); $\mathrm{t}_{\mathrm{r}}=$ 14.11 and $19.05 \mathrm{~min}$.

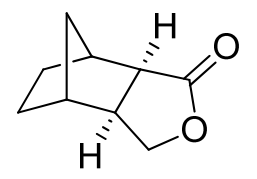

(3aS,4R,7S,7aR)-hexahydro-4,7-methanoisobenzofuran-1(3H)-one ${ }^{[4]}(17)$

The title compound was prepared according to the general procedure as described above in $>99 \%$ conversion, 92\% yield. ${ }^{1} \mathrm{H}$ NMR ( $\mathrm{CDCl}_{3}$, TMS, $\left.400 \mathrm{MHz}\right) \delta$ 4.32-4.22 (m, 2H), 3.00-2.95 (m, 1H), 2.90-2.86 (m, 1H), 2.67-2.66 (m, 1H), 2.37-2.36 (m, 1H), 1.58-1.48 (m, 6H); ${ }^{13} \mathrm{C}$ NMR $\left(\mathrm{CDCl}_{3}, \mathrm{TMS}, 100 \mathrm{MHz}\right) \delta 177.69$, 67.39, 45.76, 41.00, 40.94, 39.43, 38.86, 24.45, 20.57; The product was analyzed by GC to determine the enantiomeric excess: 87\% ee (Beta Dex 225 column, $150{ }^{\circ} \mathrm{C}, 1.0 \mathrm{~mL} / \mathrm{min}$,); $\mathrm{t}_{\mathrm{r}}=35.75$ and $37.27 \mathrm{~min}$.

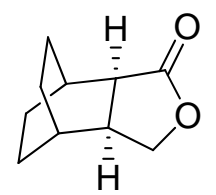

\section{(3aS,4S,7R,7aR)-hexahydro-4,7-ethanoisobenzofuran-1(3H)-one ${ }^{[5]}$ (19)}

The title compound was prepared according to the general procedure as described above in $>99 \%$ conversion, 98\% yield. ${ }^{1} \mathrm{H}$ NMR $\left(\mathrm{CDCl}_{3}\right.$, TMS, $\left.400 \mathrm{MHz}\right) \delta 4.44$ (dd, $\left.J=8.8 \mathrm{~Hz}, 16.8 \mathrm{~Hz}, 1 \mathrm{H}\right)$, 4.20 (dd, $J=3.2 \mathrm{~Hz}, 9.6 \mathrm{~Hz}, 1 \mathrm{H}), 2.68-2.66$ (m, 2H), 2.07-2.06 (m, 1H), 1.71-1.53 (m, 9H); ${ }^{13} \mathrm{C}$ NMR (CDCl 3 , TMS, 100 MHz) $\delta$ 179.88, 70.92, 41.78, 36.24, 27.09, 25.69, 24.85, 24.56, 21.60, 19.68; The product was analyzed by GC to determine the enantiomeric excess: 99\% ee (Beta Dex 120 column, $170{ }^{\circ} \mathrm{C}, 1.0 \mathrm{~mL} / \mathrm{min}$,); $\mathrm{t}_{\mathrm{r}}=14.80$ and $19.52 \mathrm{~min}$. 


\section{References:}

[1] a) H. F. Dai, W. X. Chen, L. Zhao, F. Xiong, H. Sheng, F. E. Chen, Adv. Synth. Catal., 2008, 350, 1635-1641; b) S. X. Wang, F. E. Chen, Adv. Synth. Catal. 2009, 351, 547-552.

[2] a) K. Matsuki, H. Inoue, A. Ishida, M. Takeda, M. Nakagawa, T. Hino, Chem. Pharm. Bull. 1994, 42, 9-18; b) M. D. Barker, R. A. Dixon, S. Jones, B. J. Marshc, Tetrahedron. 2006, 62, 11663-11669.

[3] I. J. Jakovac, H. B. Goodbrand, K. P Lok, J. B. Jones, J. Am. Chem. Soc. 1982, 104, 4659.

[4] P. Metz, Tetrahedron, 1989, 45, 7311-7316.

[5] P. Canonne, M. Akssira, Tetrahedron. 1985, 41, 3695-3704. 


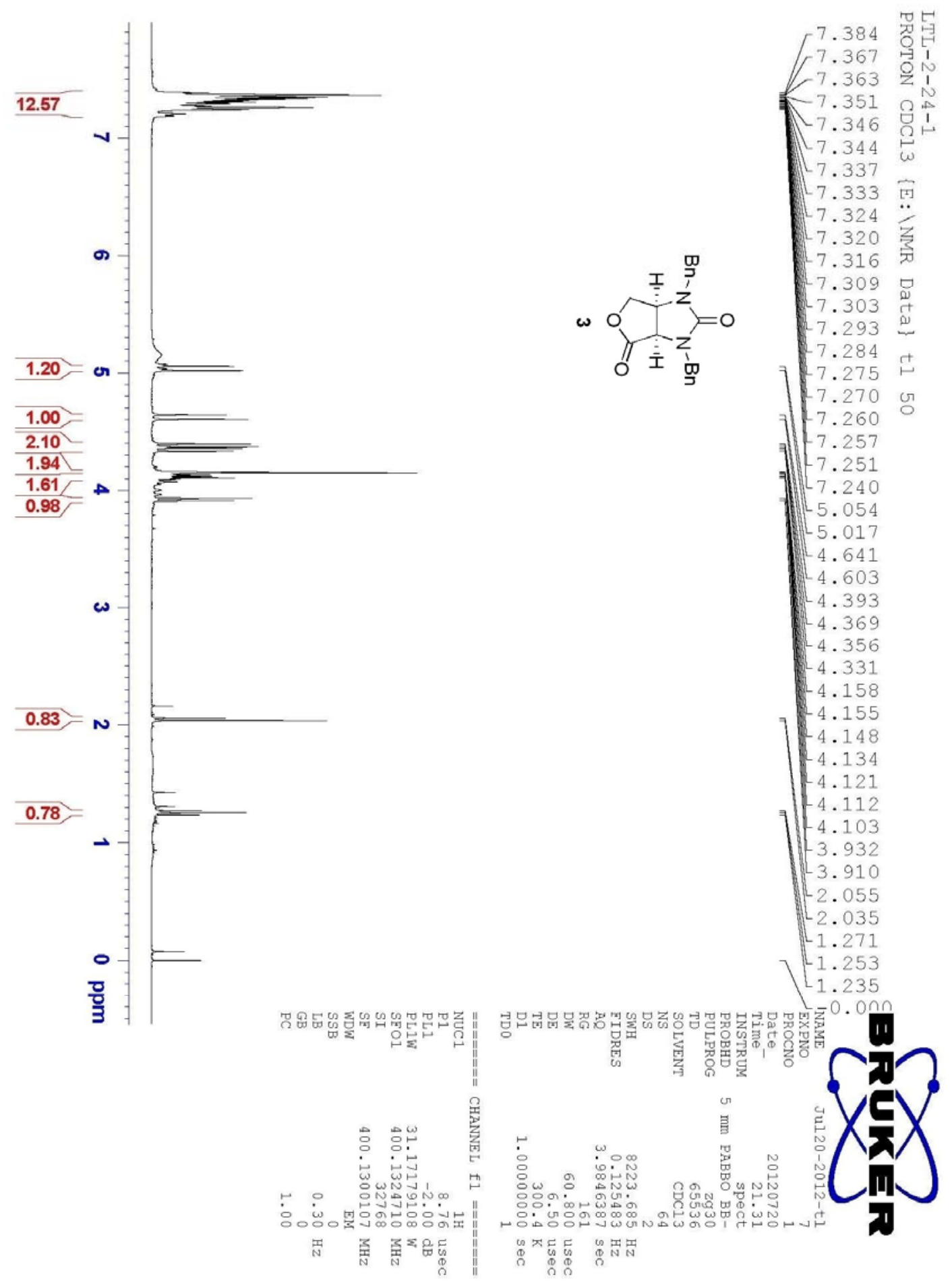




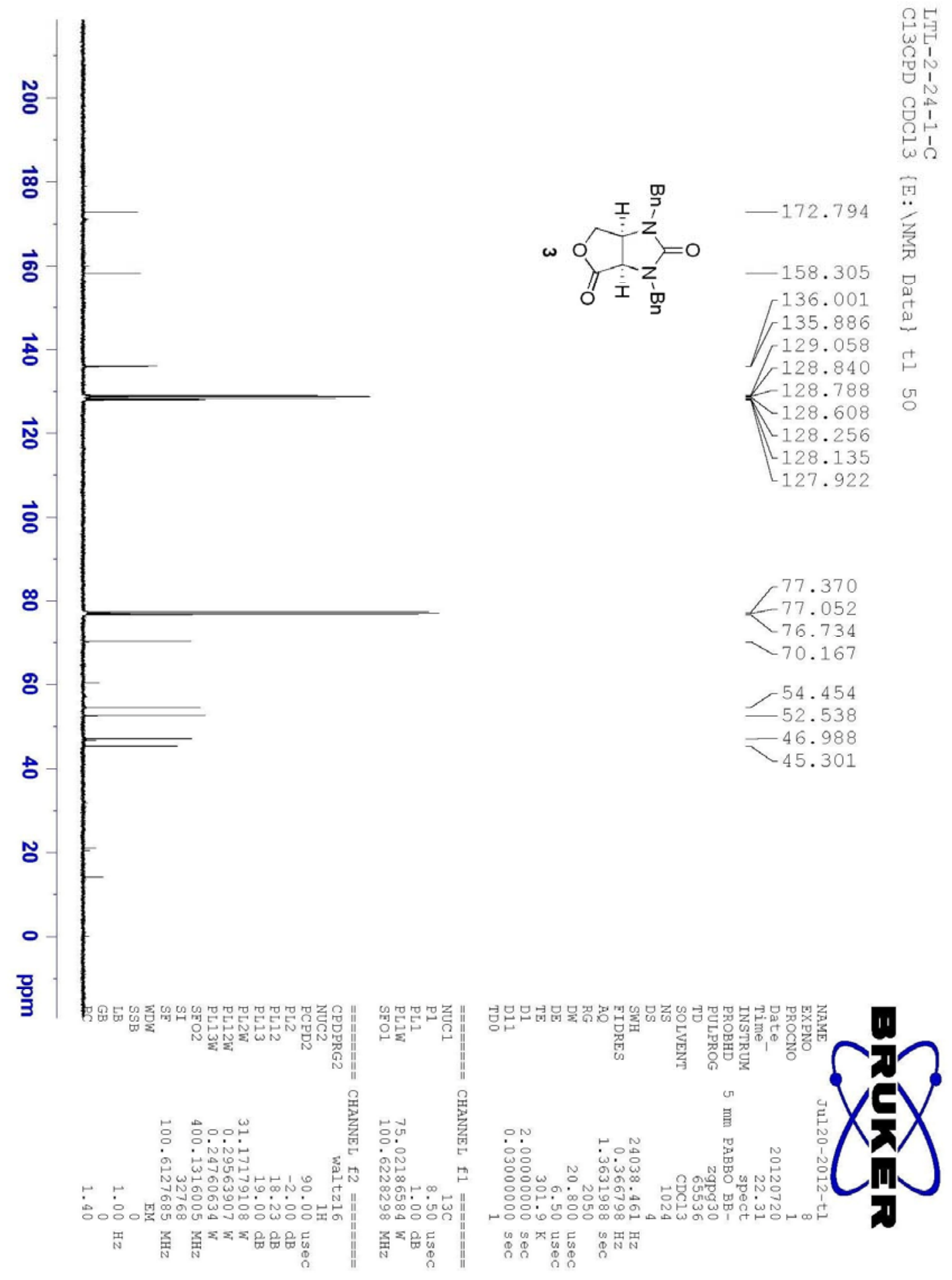




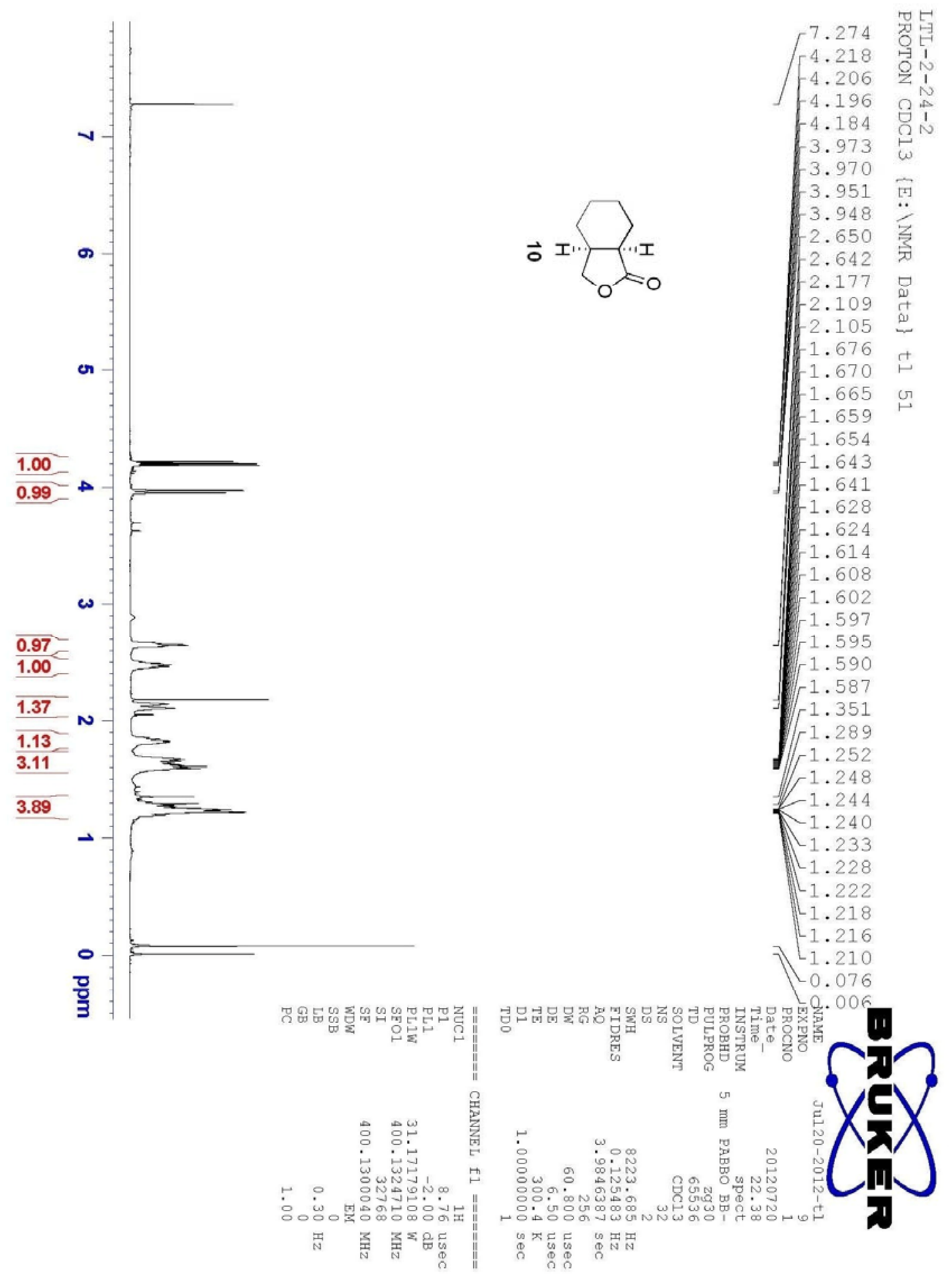




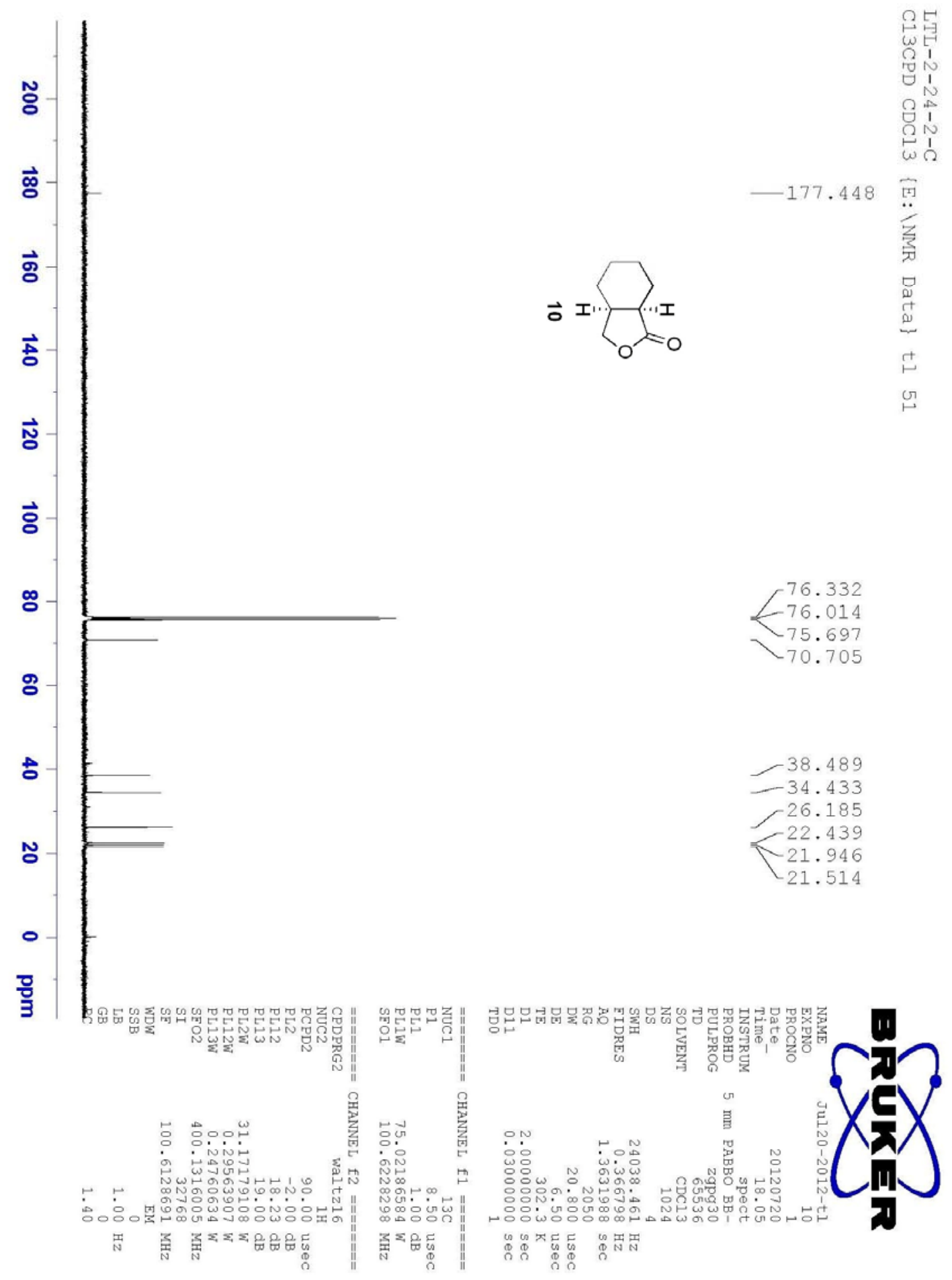



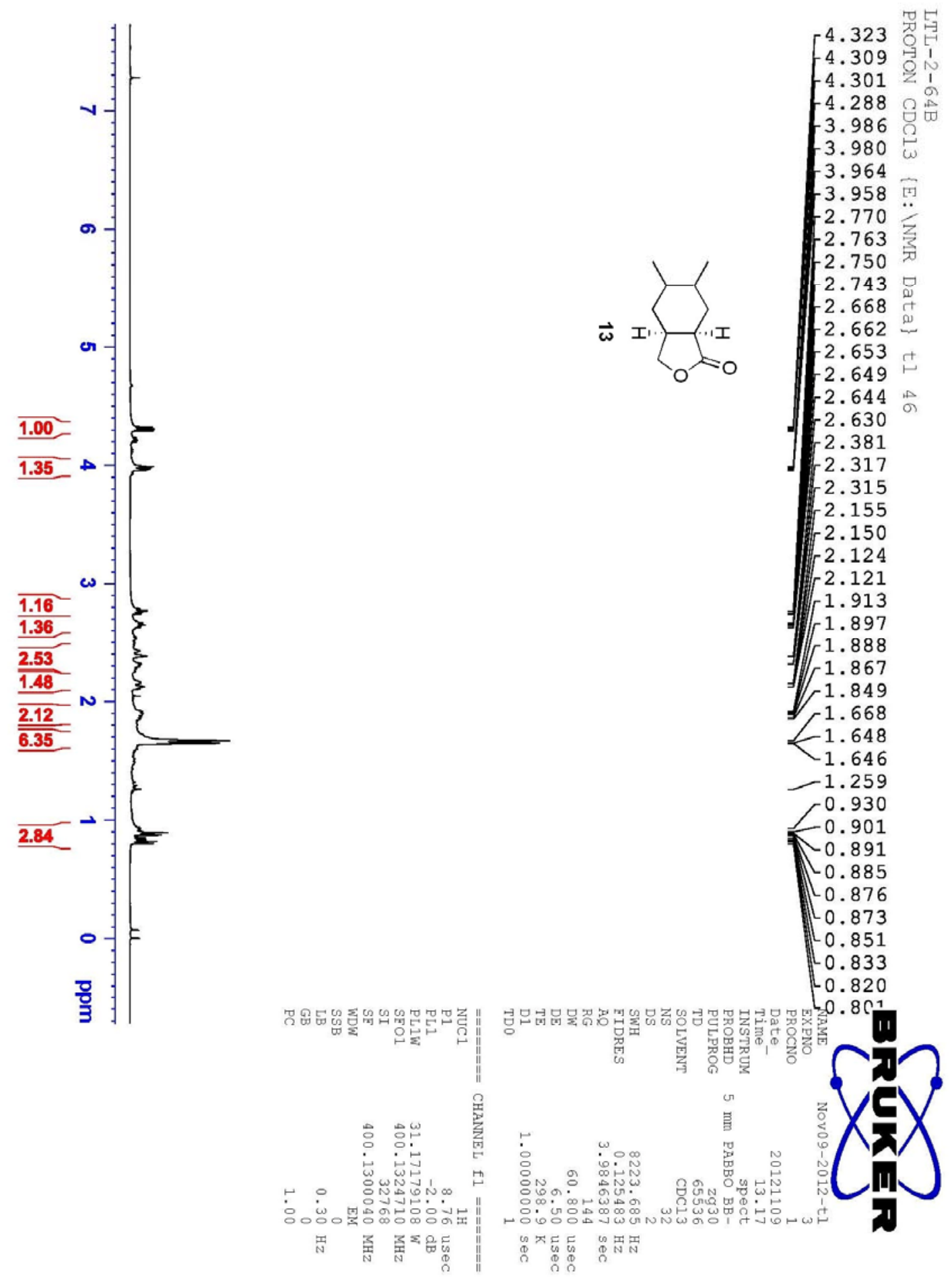


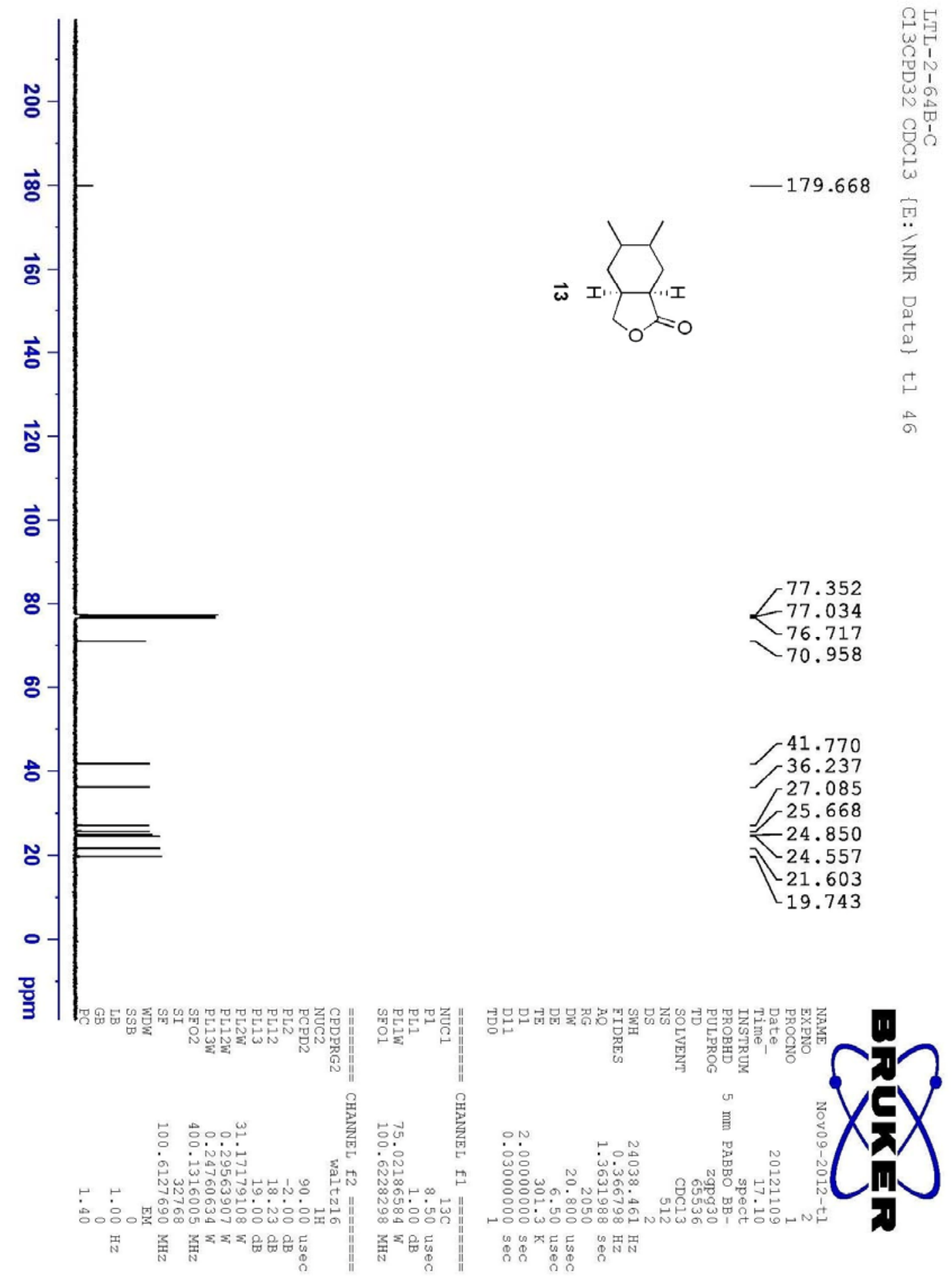



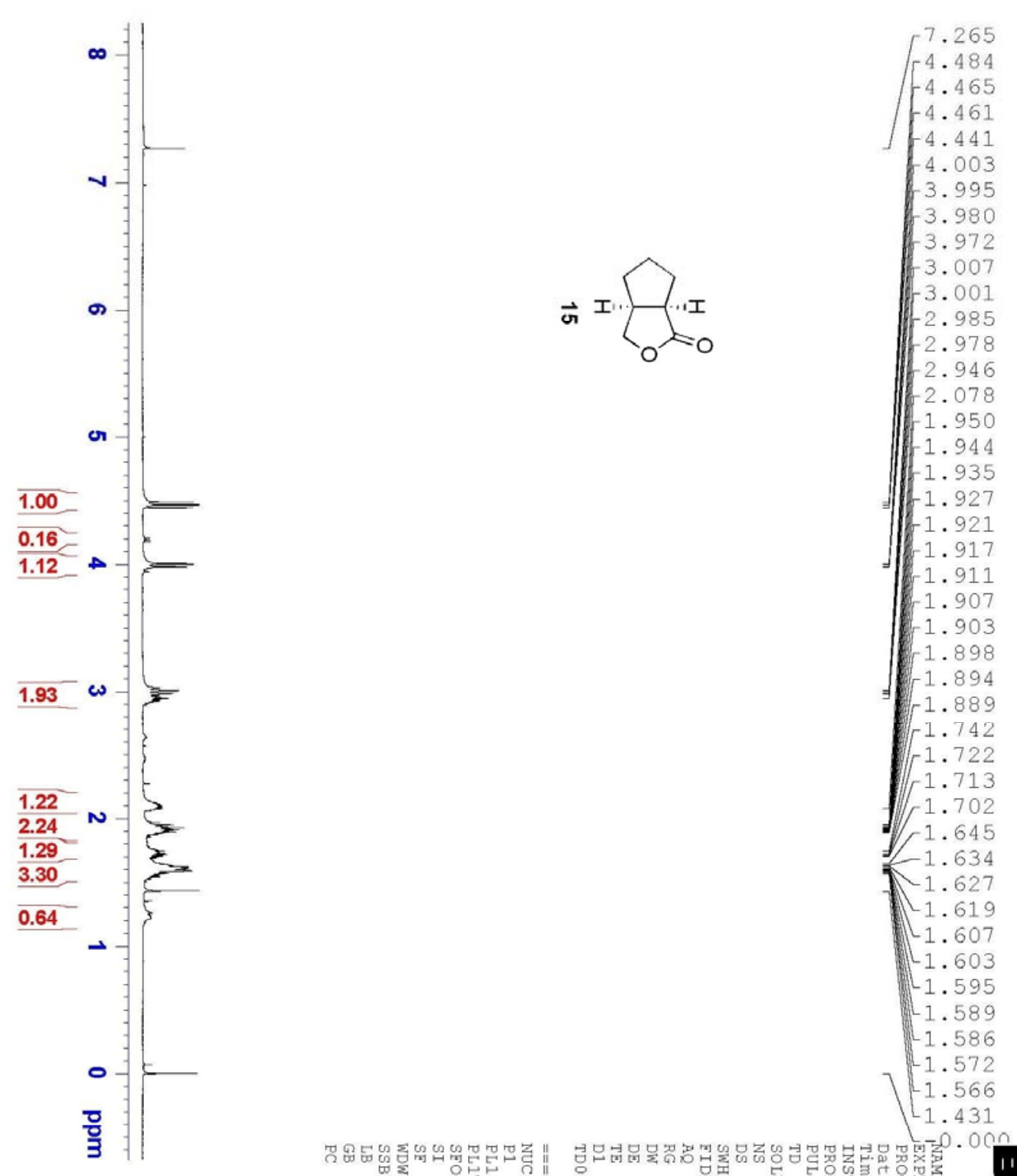

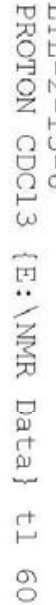

-1.944 앙

-1.927

$-1.921$

$-1.917$

$-1.911$

1.907

$-1.903$

1.898
-1.894

1.889

$-1.742$

$-1.722$

. 1.713

1.702

1.645

$-1.634$

$-1.627$

1.619

$-1.607$

$-1.603$

$-1.595$

$-1.589$

$-1.586$

1.572

1.566

1.431

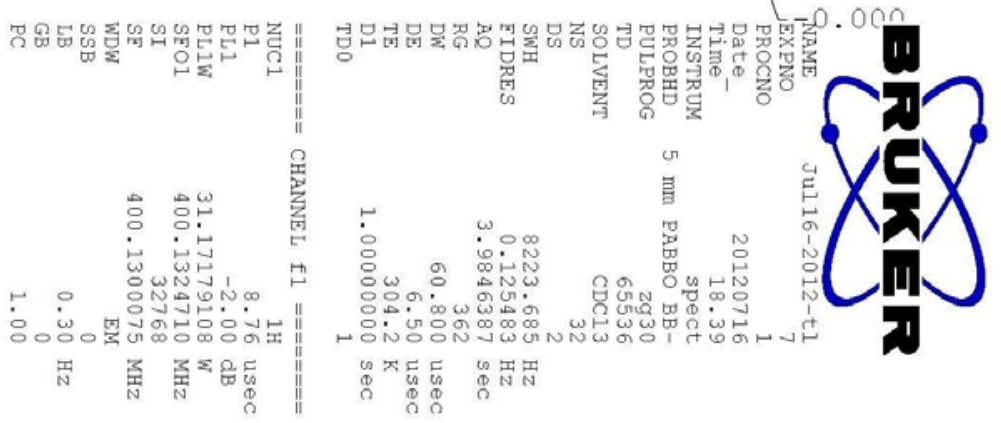




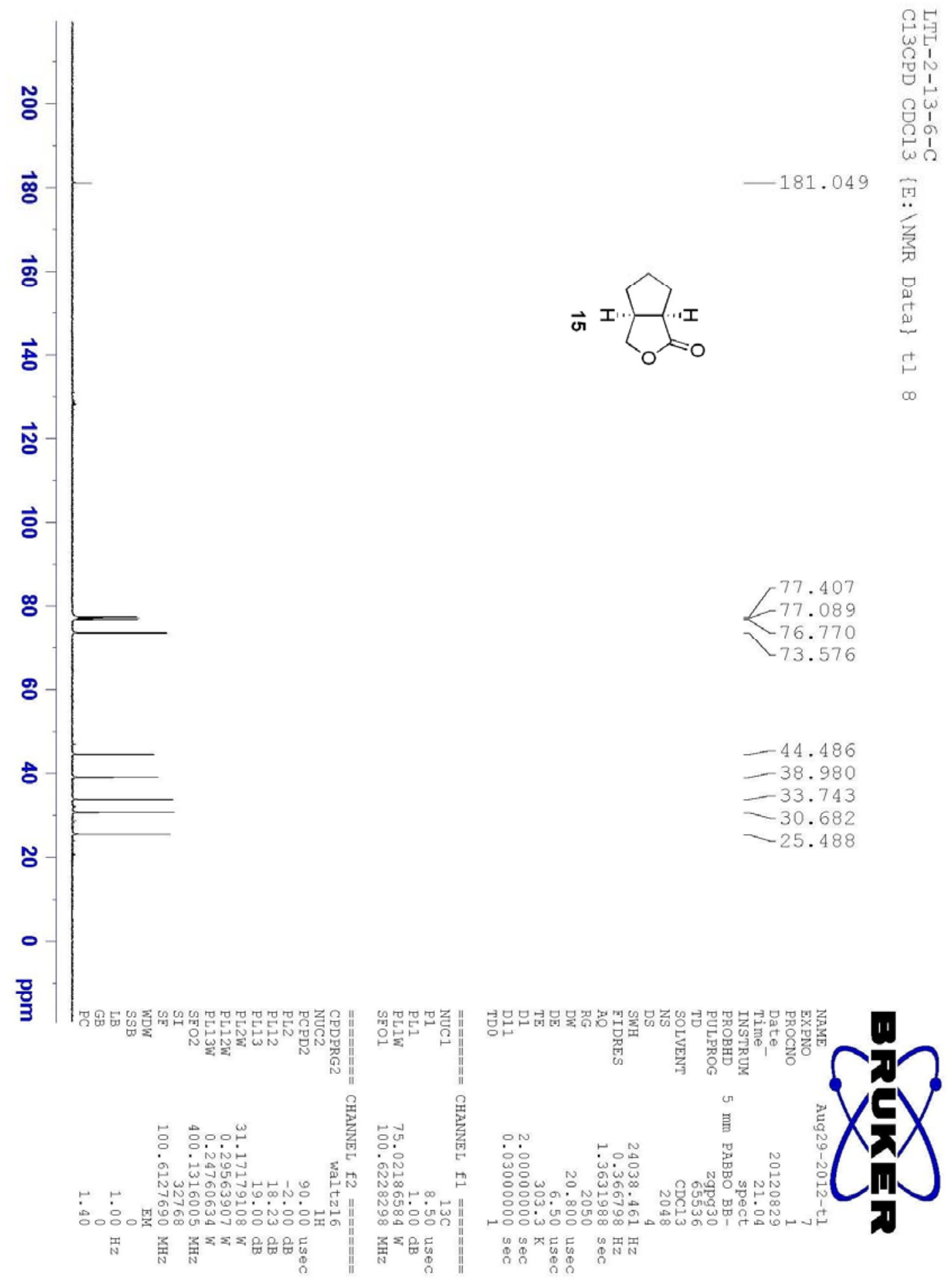




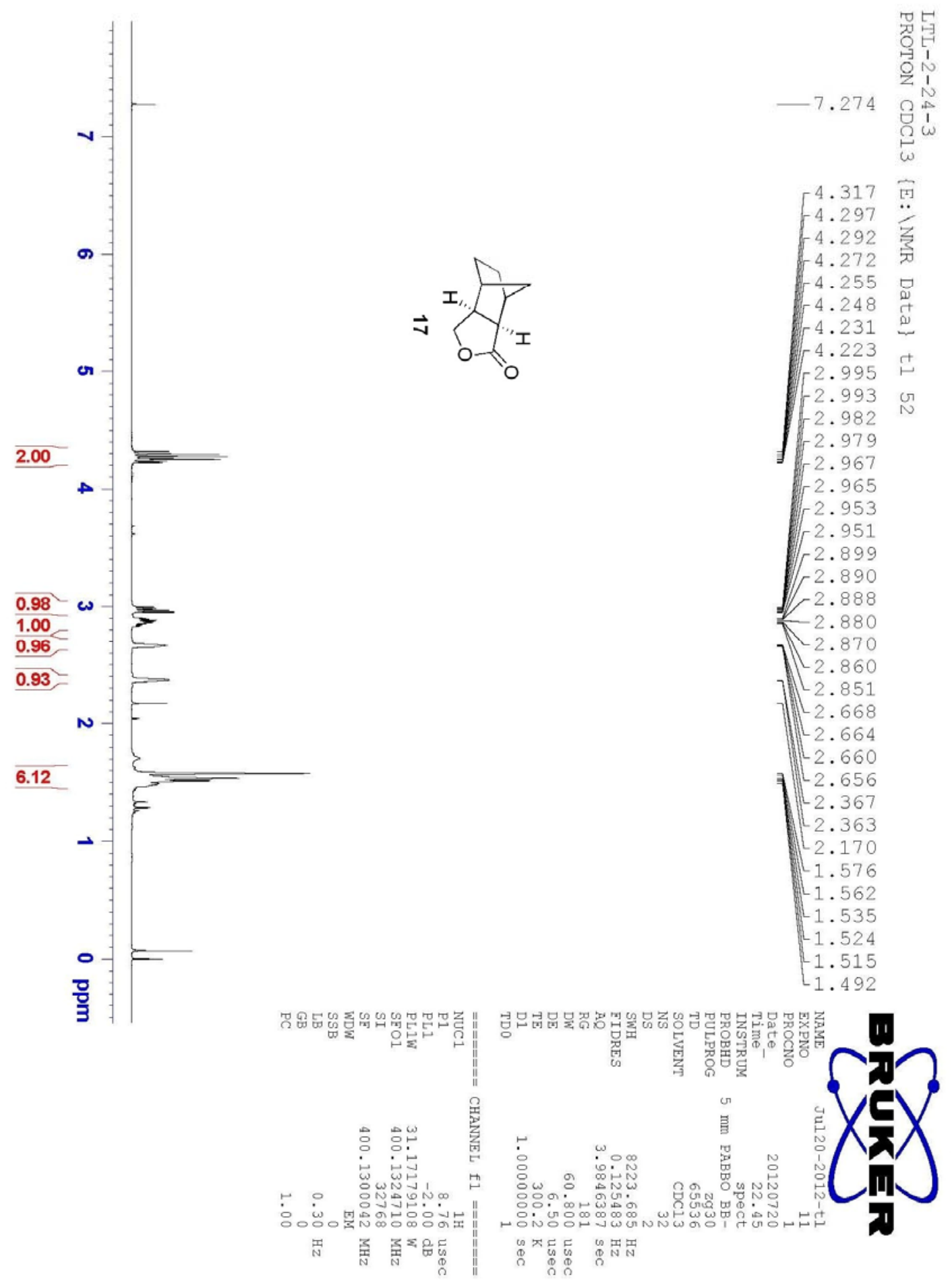




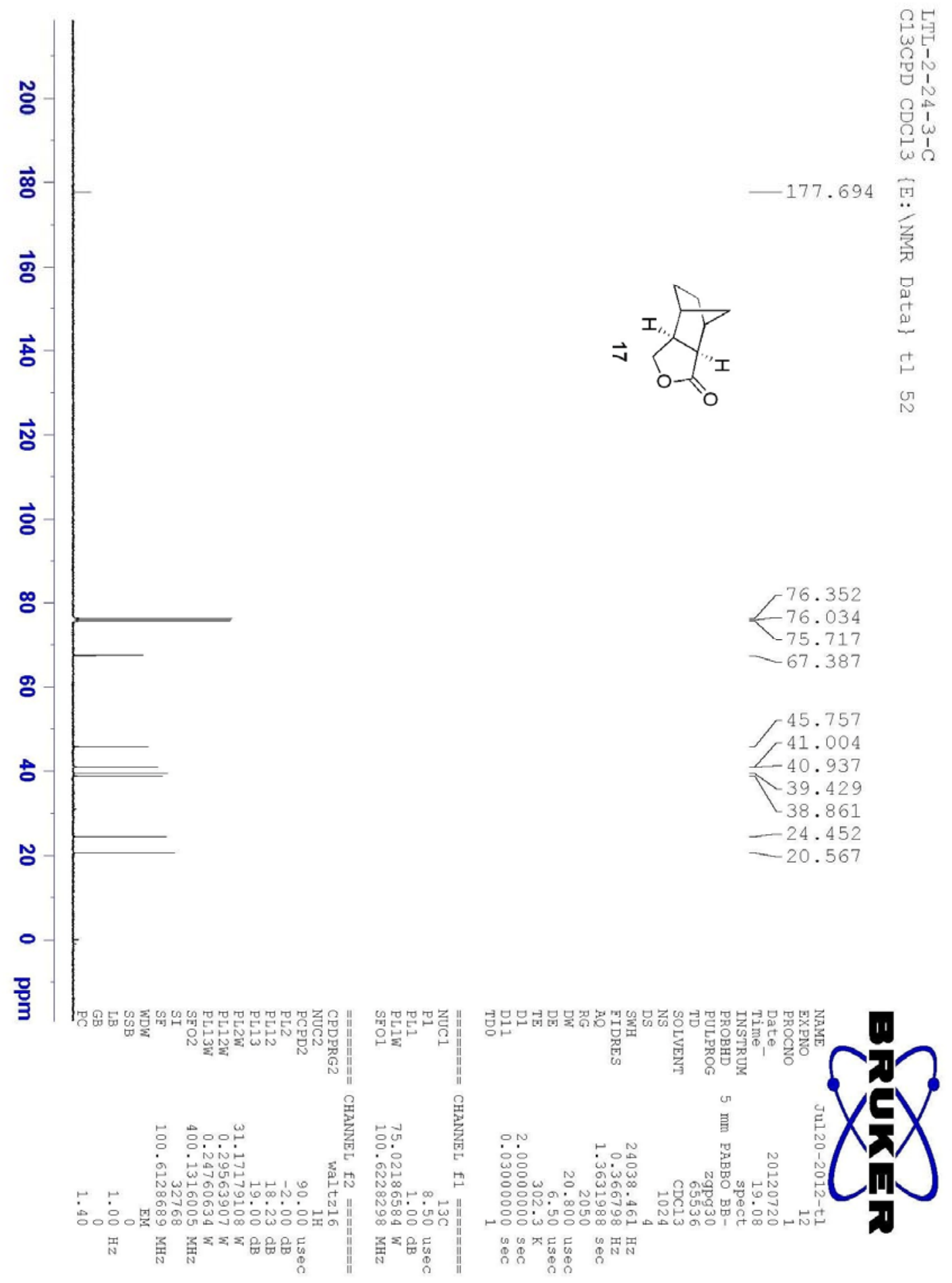



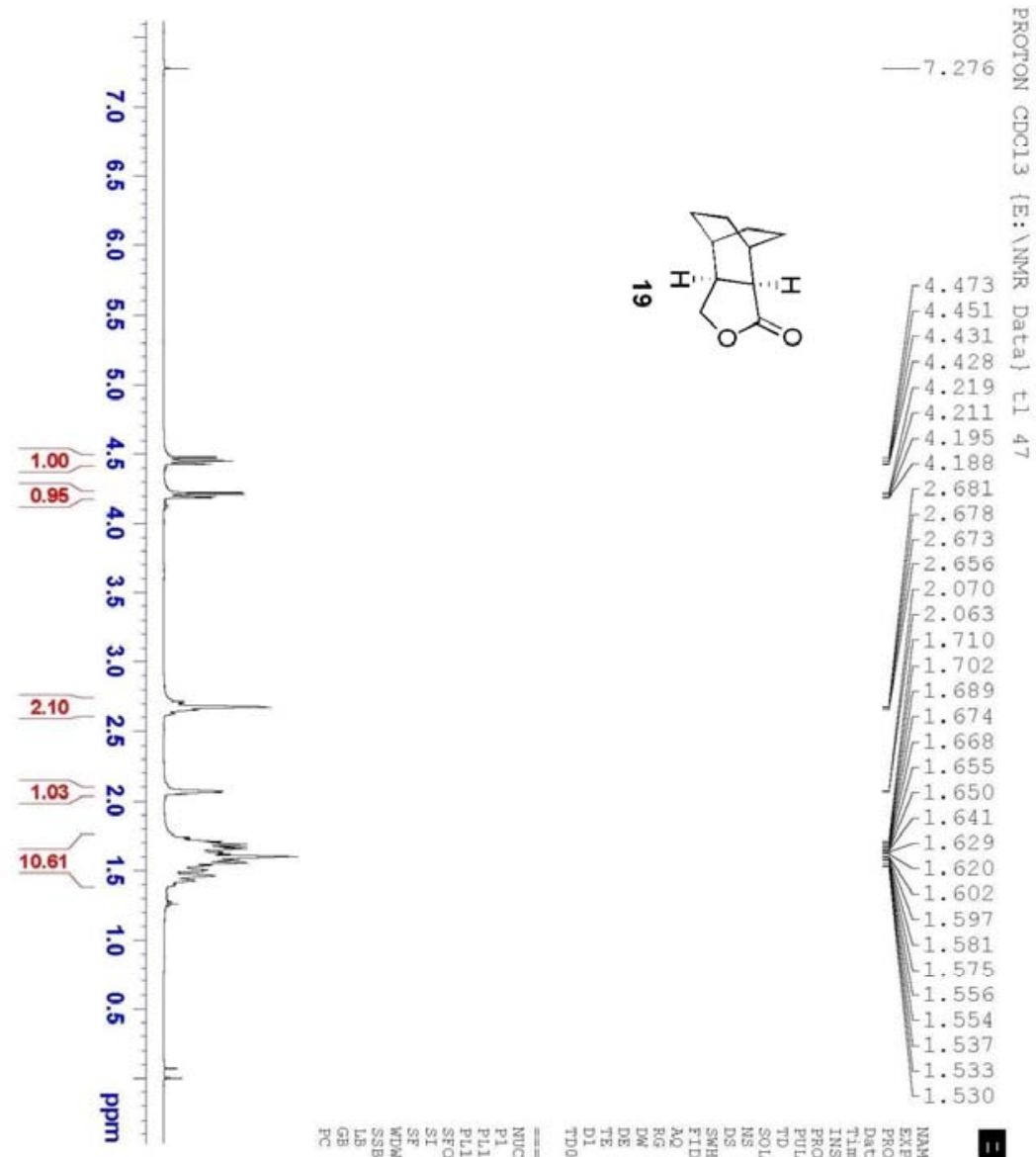

I 2.681

$-2.678$

2.673
-2.656

$\left[\begin{array}{r}2.056 \\ -2.070\end{array}\right.$

2.063

-2.063
-1.710

$-1.702$

$\left[\begin{array}{r}1.674 \\ -1.668\end{array}\right.$

$\left[\begin{array}{l}1.674 \\ 1.668\end{array}\right.$

$\left[\begin{array}{l}1.668 \\ 1.655\end{array}\right.$

$\left.\int\right]^{1.650}$

1.641

1.620

$L_{1.602}^{1.620}$

$L_{1.597}^{-58}$

$\left\{\begin{array}{l}1.575 \\ 1.556\end{array}\right.$

1.556
1.554

$-1.554$

-1.537
-1.533

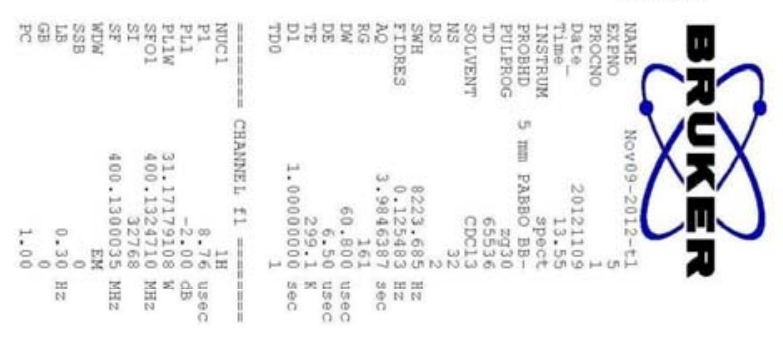




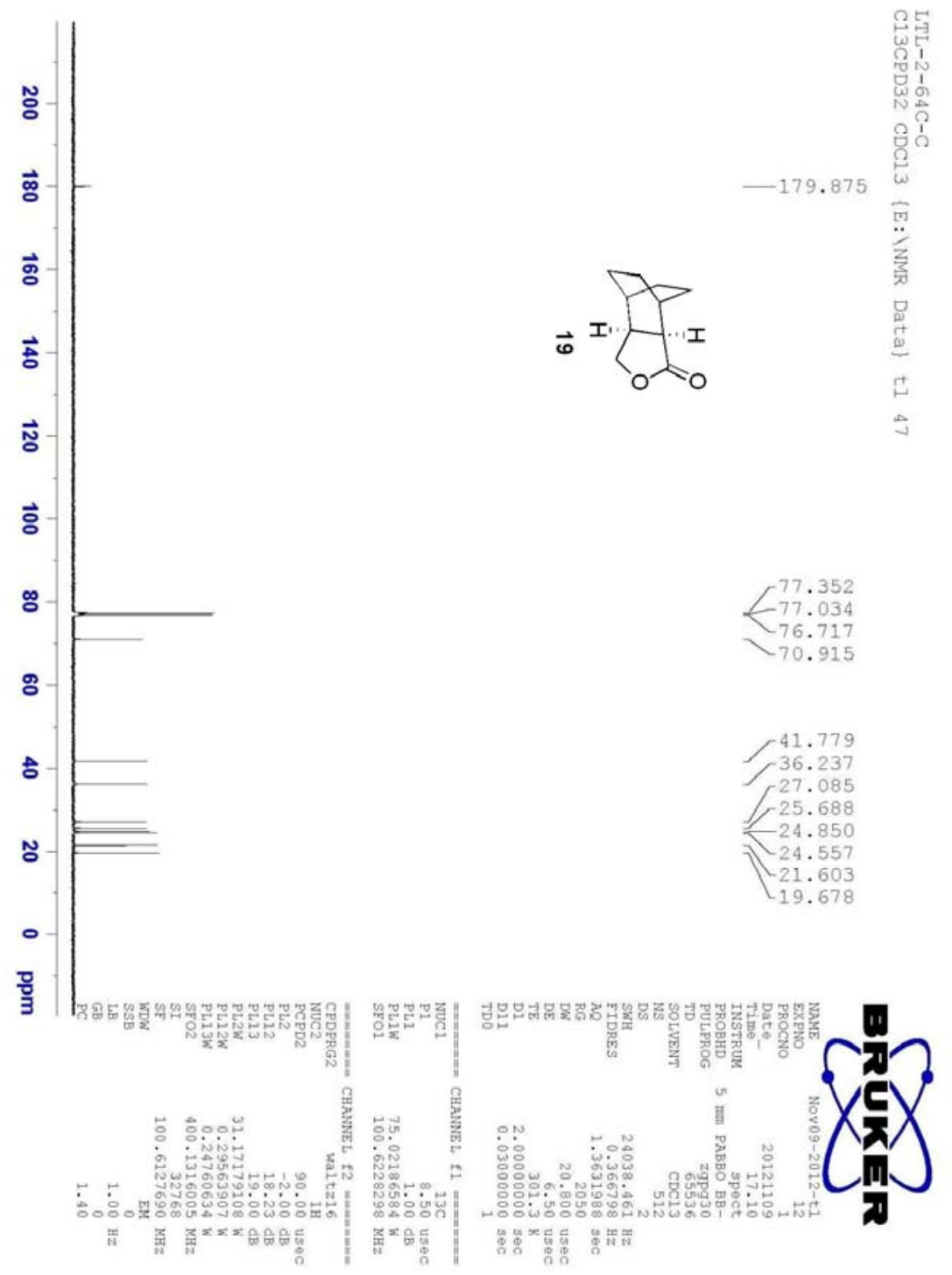


Data File C: \CHEM32 $\backslash 1 \backslash$ DATA $\backslash L T L \backslash L T L-13-1000001 . D$ Sample Name: LTL-13-1
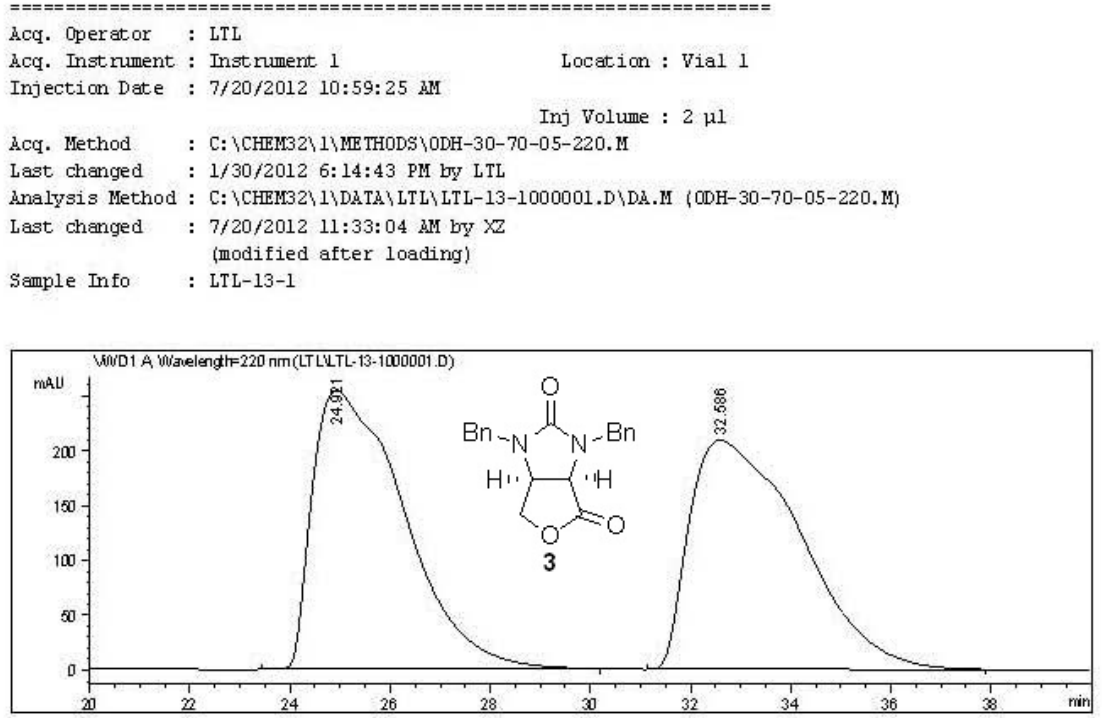

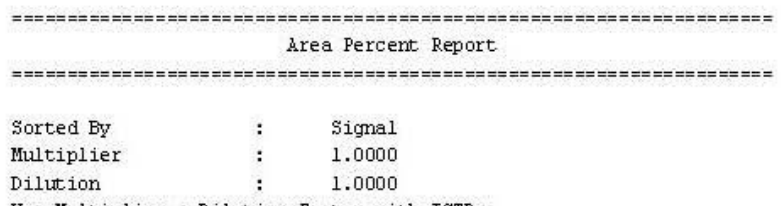

Use Multiplier \& Dilution Factor with IsTDs

Signal 1: VTD $1 \mathrm{~A}$, Wavelength $=220 \mathrm{~nm}$

\begin{tabular}{|c|c|c|c|c|c|}
\hline \multirow{2}{*}{$\begin{array}{c}\text { Peak } \\
\# \\
-1\end{array}$} & \multirow{2}{*}{$\begin{array}{l}\text { RetTime Type } \\
\text { [min] }\end{array}$} & \multirow{2}{*}{$\begin{array}{l}\text { Width } \\
\text { [min] }\end{array}$} & Area & Height & \multirow{2}{*}{$\begin{array}{c}\text { Area } \\
\text { a }\end{array}$} \\
\hline & & & madU & [m.3JJ & \\
\hline 1 & & & 3. $23398 \mathrm{e} 4$ & 254.3 & \\
\hline 2 & $32.586 \mathrm{BB}$ & 594 & $3.22630 \mathrm{e} 4$ & 208.99203 & 49.9405 \\
\hline 1000 & & & $6.46028 \mathrm{e} 4$ & 463.33913 & \\
\hline
\end{tabular}

*** End of Report *t* 
Data File C: \CHEM32 $\backslash 1 \backslash$ DATA \LTL $\backslash L T L-2-24-100001 . D$

Sample Name: LTL-2-24-1
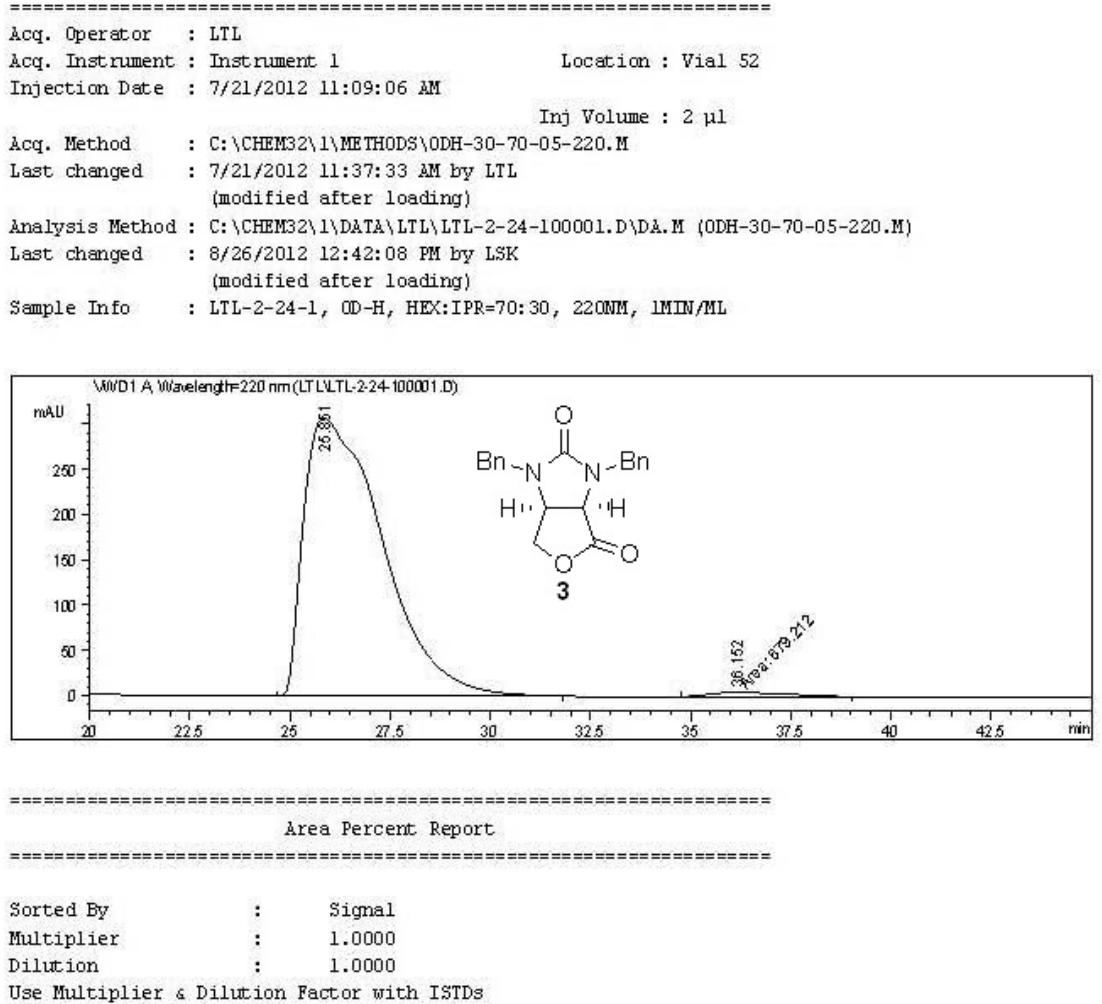

Signal 1: VID $1 \mathrm{~A}$, Wavelength $=220 \mathrm{~nm}$

\begin{tabular}{|c|c|c|c|c|c|c|}
\hline Peak & RetTime Type & Width & & rea & Height & Area \\
\hline$\#$ & [min] & [min] & $\mathrm{mBU}$ & $*_{3}$ & {$[\mathrm{~m} \mathrm{~m} \mathrm{BJ}$} & 흡 \\
\hline 1 & $25.851 \mathrm{BB}$ & 1.8795 & 4.17 & $840 \mathrm{e} 4$ & 306.63101 & 98.4005 \\
\hline 2 & $36.152 \mathrm{MM}$ & 2.2079 & 679 & .21191 & 5.12711 & 1.5995 \\
\hline Tulal & Is: & & 4.24 & $632 \mathrm{e} 4$ & 311.75612 & \\
\hline
\end{tabular}


Data File C: \CHEM32 $\backslash \backslash$ DATA $\backslash L T L \backslash L T L-2-13-200001 . D$

Sample Name: LTL $-2-13-2$
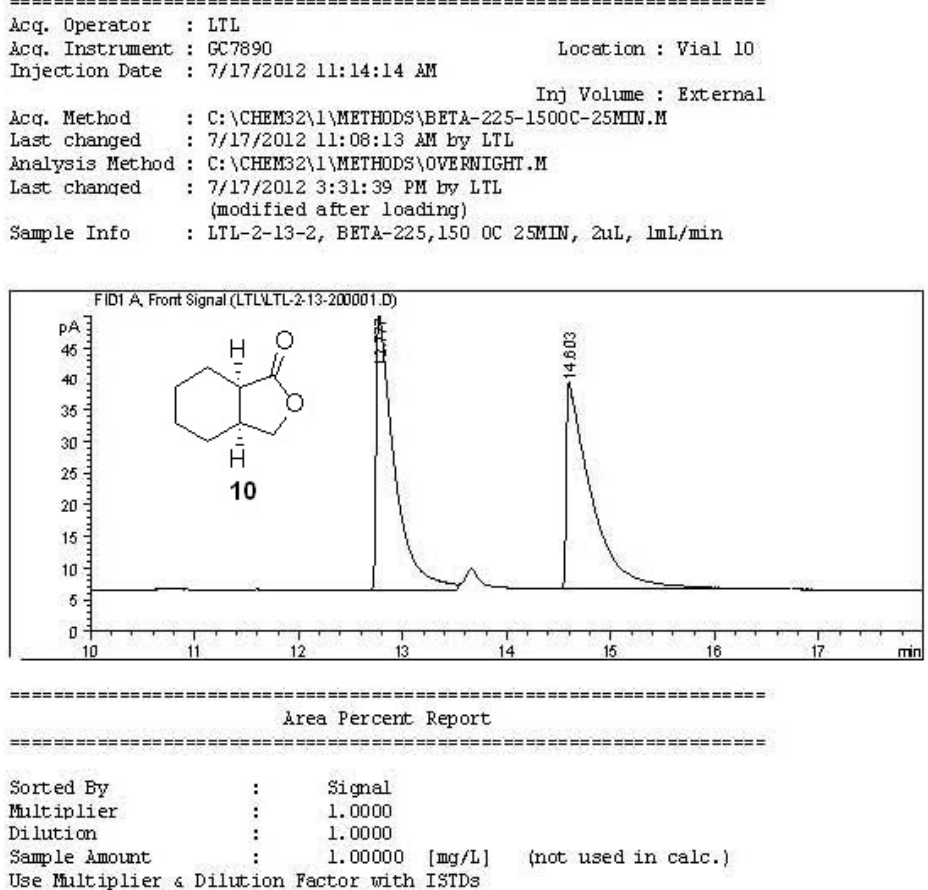

Jample Amount

Signal 1: FIDI A, Front Sigmal

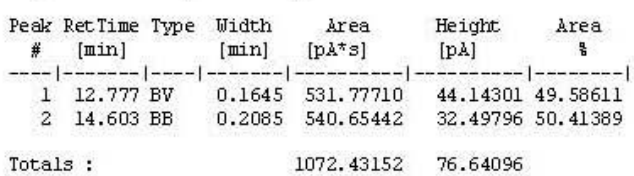

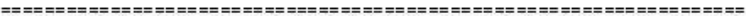

桀 End of Report 
Data File C: \CHEM32 $\backslash \backslash$ DATA $\backslash L T L \backslash L T L-2-29-300001 . D$

Sample Name: LTL $-2-29-3$
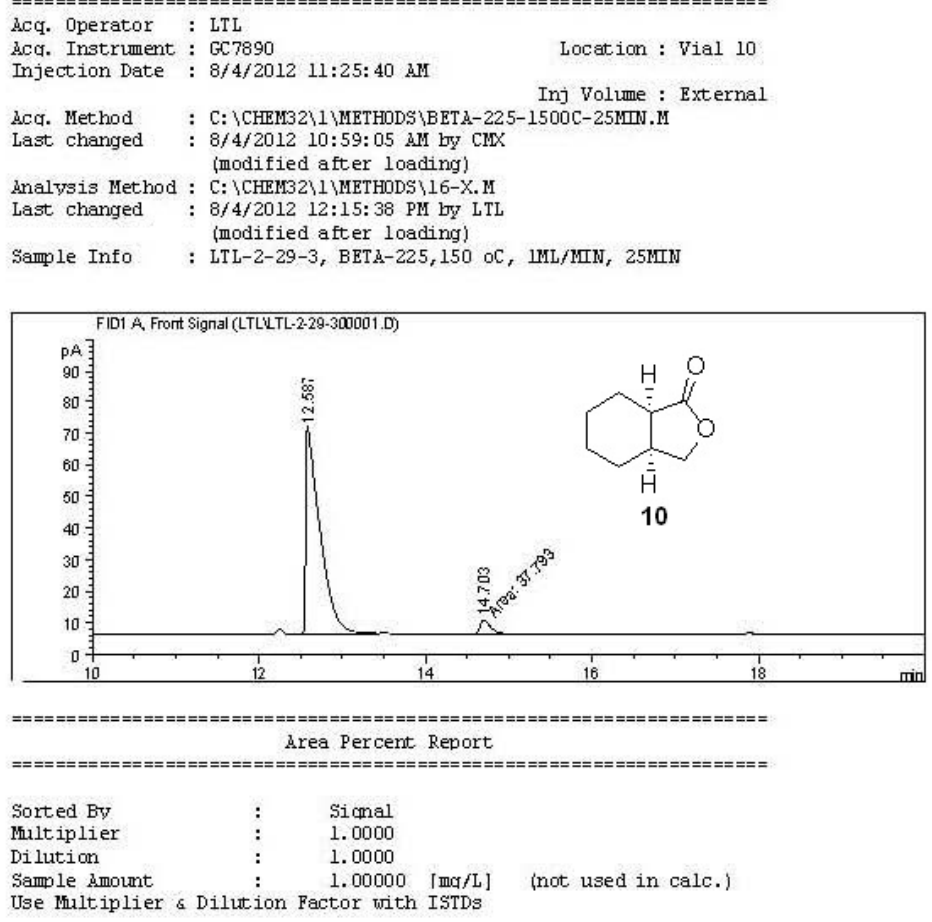

Use Pultiplier \& Dilution Factor with ISTD

Signal 1: FID1 A, Front Signal

\begin{tabular}{|c|c|c|c|c|c|c|}
\hline $\begin{array}{c}\text { Peak } \\
\#\end{array}$ & $\begin{array}{l}\text { Ret Time } \\
\text { [min] }\end{array}$ & Type & $\begin{array}{l}\text { Width } \\
\text { [min] }\end{array}$ & $\begin{array}{r}\text { Area } \\
\left.\mid \mathrm{p} A^{*} s\right]\end{array}$ & $\begin{array}{l}\text { Height } \\
\text { [DA] }\end{array}$ & $\underset{\substack{\text { Area } \\
\mathrm{a}}}{\mathrm{a}}$ \\
\hline 1 & 12.587 & $\mathrm{BV}$ & 0.1567 & 783. & 64. & \\
\hline 2 & 14.703 & $\mathrm{MIM}$ & 0.1438 & 37.79303 & 4.38119 & 4.60006 \\
\hline & & & & 821.57727 & 69.36623 & \\
\hline
\end{tabular}

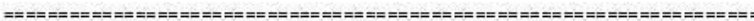

t大t End of Report 
Data File C: $\backslash$ CHEM $32 \backslash 1 \backslash$ DATA $\backslash L T L \backslash L T L-2-62 B 000050 . D$ Sample Name: LTL-2-62B
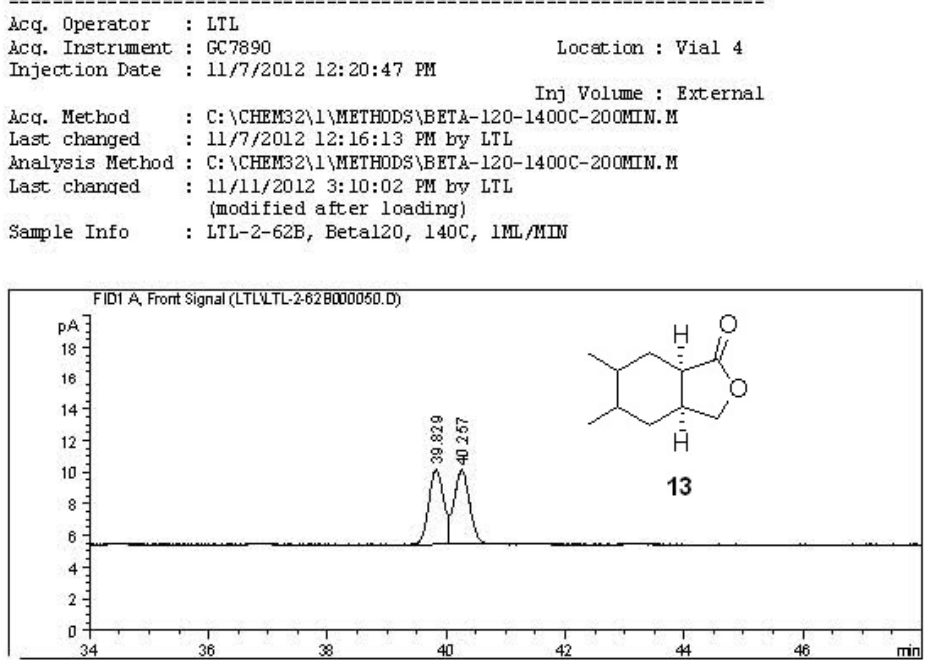

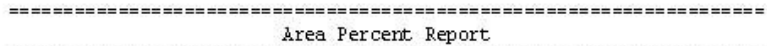

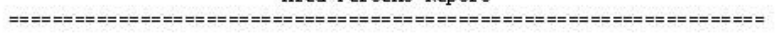

Sorted By

Philtiplier

Dilution

: Signal

$\vdots \quad 1.0000$

$: \quad 1.00000 \quad[\mathrm{mg} / \mathrm{L}]$ (not used in calc.)

Use liultiplier \& Dilution Factor with IsTDs

Signal 1: FIDl A, Front Sigmal

\begin{tabular}{|c|c|c|c|c|c|c|}
\hline $\begin{array}{c}\text { Peakz } \\
\#\end{array}$ & $\begin{array}{l}\text { Ret Time } \\
\text { [min] }\end{array}$ & Type & $\begin{array}{l}\text { Width } \\
\text { [min] }\end{array}$ & $\begin{array}{r}\text { Area } \\
{\left[\mathrm{pA}^{*} \mathrm{~s}\right]}\end{array}$ & $\begin{array}{l}\text { Height } \\
{[\mathrm{pA}]}\end{array}$ & $\underset{\substack{\text { A } \\
\text { A }}}{ }$ \\
\hline 1 & 39.829 & BV & 0.2655 & 82.67277 & 4948 & 02 \\
\hline 2 & 40.257 & VB & 0.2783 & 84.75890 & 4.68497 & 50.62298 \\
\hline tal & & & & 7.43166 & 9.43445 & \\
\hline
\end{tabular}

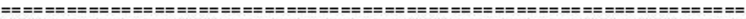

桀 End of Report 
Data File C: $\backslash$ CHEM $32 \backslash 1 \backslash$ DATA $\backslash L T L \backslash L T L-2-64$ B000055.D

Sample Name: LTL-2-64B
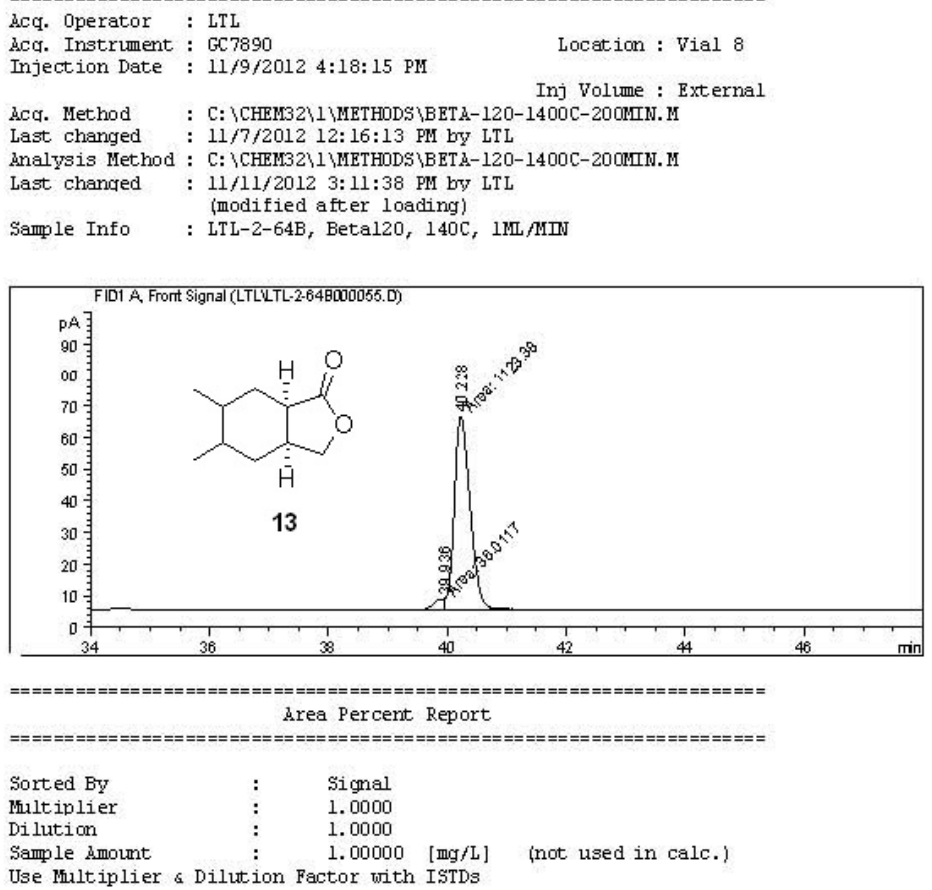

Use Phltiplier \& Dilution Factor with IsTDs

Signal 1: FIDl A, Front Signal

\begin{tabular}{|c|c|c|c|c|c|c|}
\hline $\begin{array}{c}\text { Peakz } \\
\#\end{array}$ & $\begin{array}{l}\text { Ret Time } \\
\text { [min] }\end{array}$ & Type & $\begin{array}{l}\text { Width } \\
\text { [min] }\end{array}$ & $\begin{array}{r}\text { Area } \\
{\left[\mathrm{pA}^{*} \mathrm{~s}\right]}\end{array}$ & $\begin{array}{l}\text { Height } \\
\text { [pA] }\end{array}$ & $\underset{\substack{\text { An } \\
\text { Area }}}{ }$ \\
\hline 1 & & $\mathrm{MF}$ & 0. & 166 & 512 & 509 \\
\hline 2 & 40.228 & $\mathrm{FM}$ & 0.3068 & 1123.37634 & 61.03107 & 96.89391 \\
\hline & & & & 59.38800 & 64.27619 & \\
\hline
\end{tabular}

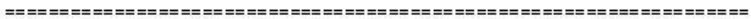

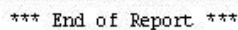


Data File C: \CHEM32 $\backslash \backslash$ DATA $\backslash L T L \backslash L T L-2-13-600009 . D$

Sample Name: LTL $-2-13-6$
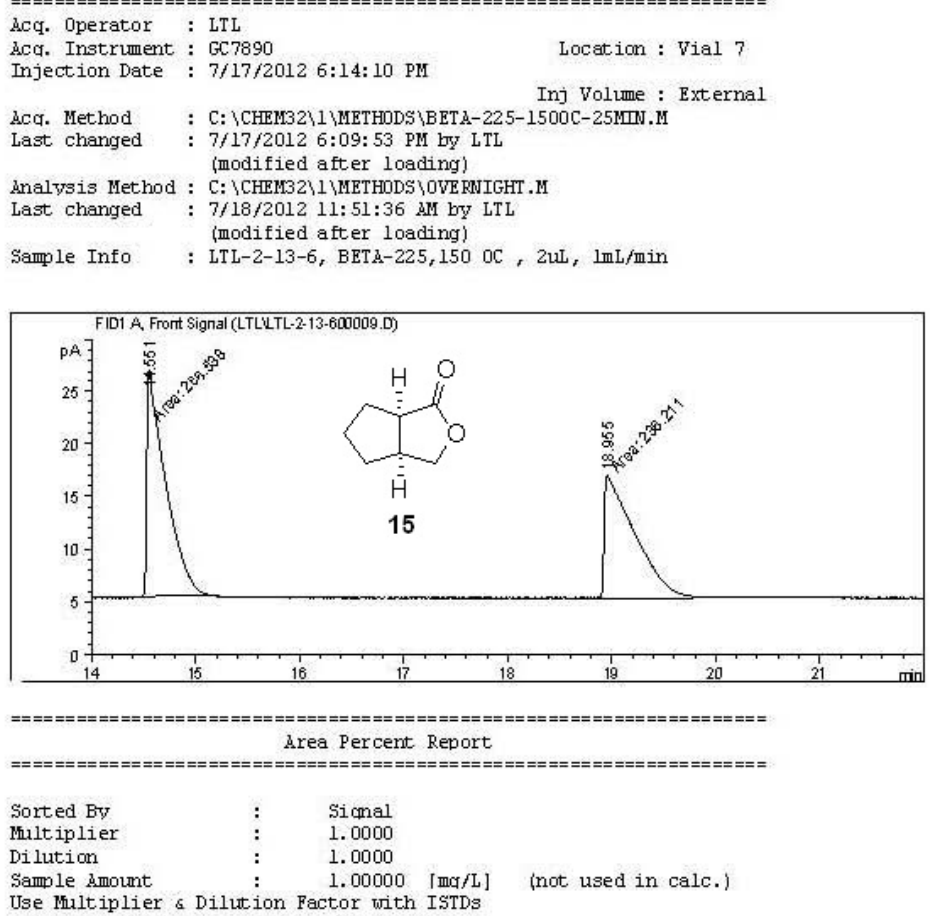

Use Pultiplier \& Dilution Factor with IsTDs

Signal 1: FID1 A, Front Signal

\begin{tabular}{|c|c|c|c|c|c|c|}
\hline eak & $\begin{array}{l}\text { Ret Time } \\
\text { [min] }\end{array}$ & Type & $\begin{array}{l}\text { Width } \\
\text { [min] }\end{array}$ & $\begin{array}{r}\text { Area } \\
\left|p A^{*} s\right|\end{array}$ & $\begin{array}{l}\text { Height } \\
{[\mathrm{DA}]}\end{array}$ & $\underset{\substack{\text { Area } \\
\frac{a}{b}}}{ }$ \\
\hline 1 & 14 & $\mathrm{M}$ & 0.2058 & 266.53815 & & 618 \\
\hline 2 & 18.955 & $\mathrm{MM}$ & 0.3375 & 236.21053 & 11.66311 & 46.98382 \\
\hline 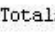 & & & & 502.74867 & 33.24788 & \\
\hline
\end{tabular}

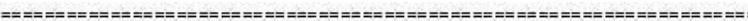

औtה End of Report 


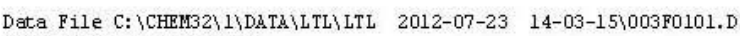
Sample Name: LTL $-2-25-5$

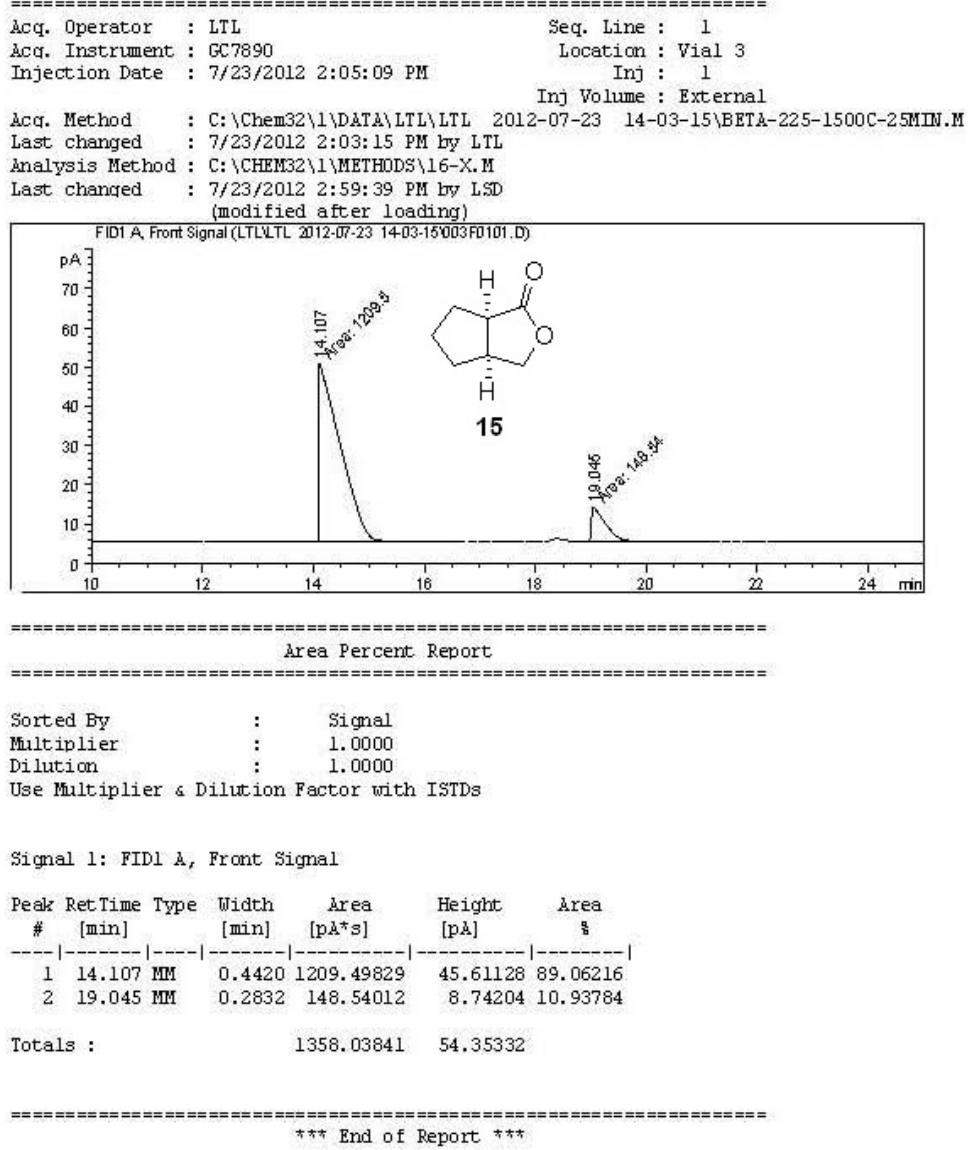


Data File C: \CHEM32 $\backslash \backslash$ DATA $\backslash L T L \backslash L T L-2-13-300006 . D$

Sample Name: LTL $-2-13-3$
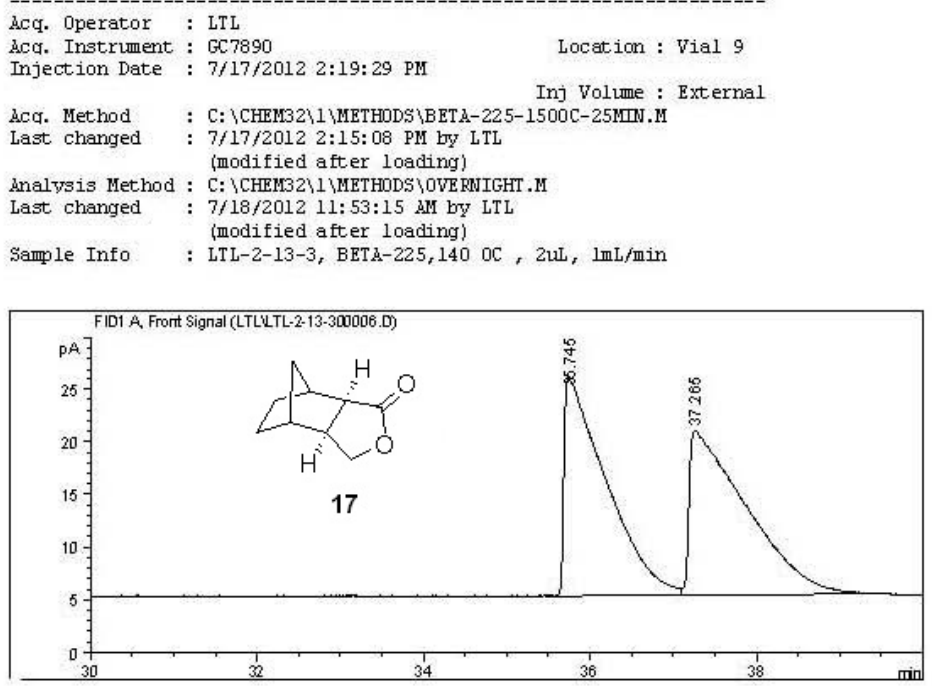

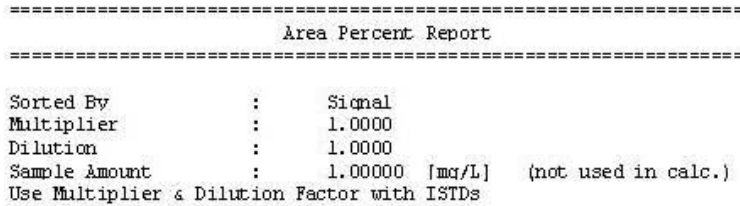

Use Phultiplier \& Dilution Factor with ISTDs

Signal 1: FID1 A, Front Signal

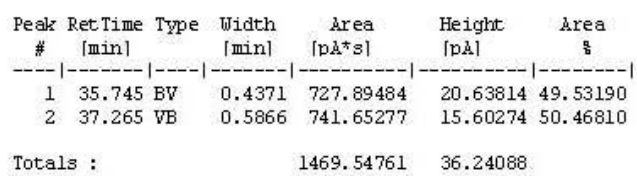

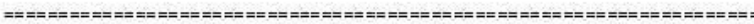

tतt End of Report 
Data File C: \CHEM32 $\backslash \backslash$ DATA $\backslash L T L \backslash L T L-2-29-200003 . D$ Sample Name: LTL $-2-29-2$
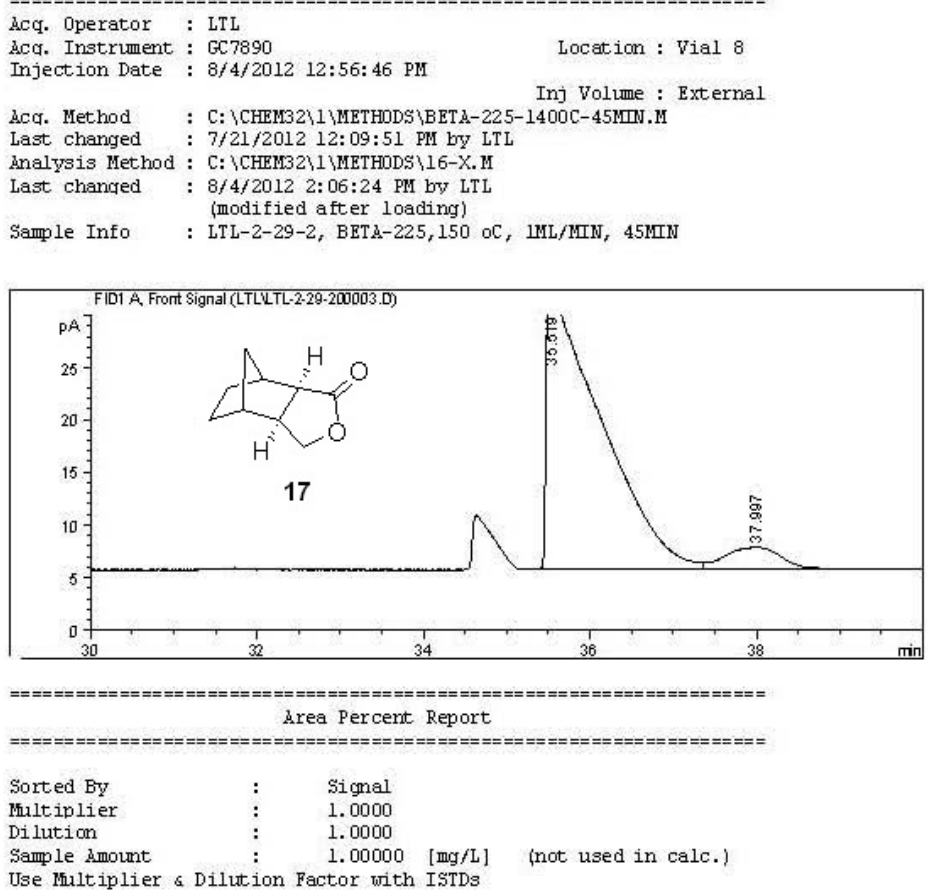

Sample amount

Signal 1: FIDI A, Front Sigmal

\begin{tabular}{|c|c|c|c|c|c|}
\hline $\begin{array}{c}\text { Peakz } \\
\#\end{array}$ & $\begin{array}{l}\text { Ret Time Type } \\
\text { [min] }\end{array}$ & $\begin{array}{l}\text { Width } \\
\text { [min] }\end{array}$ & $\begin{array}{r}\text { Area } \\
{\left[\mathrm{p}^{*} s\right]}\end{array}$ & $\begin{array}{l}\text { Height } \\
{[\mathrm{pA}]}\end{array}$ & $\underset{\substack{\text { A } \\
\text { Area }}}{ }$ \\
\hline 1 & $9 \mathrm{BV}$ & 0.5520 & 1219 & 26. & 92.47763 \\
\hline 2 & 97 VB & 0.5678 & 99.22027 & 2.07386 & 7.52237 \\
\hline & & & 1319.00249 & 28.78337 & \\
\hline
\end{tabular}

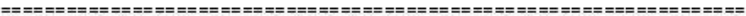

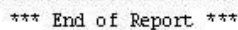


Data File C: $\backslash$ CHEM $32 \backslash 1 \backslash$ DATA $\backslash L T L \backslash L T L-2-62 C 000051 . D$ Sample Name: LTL-2-62C
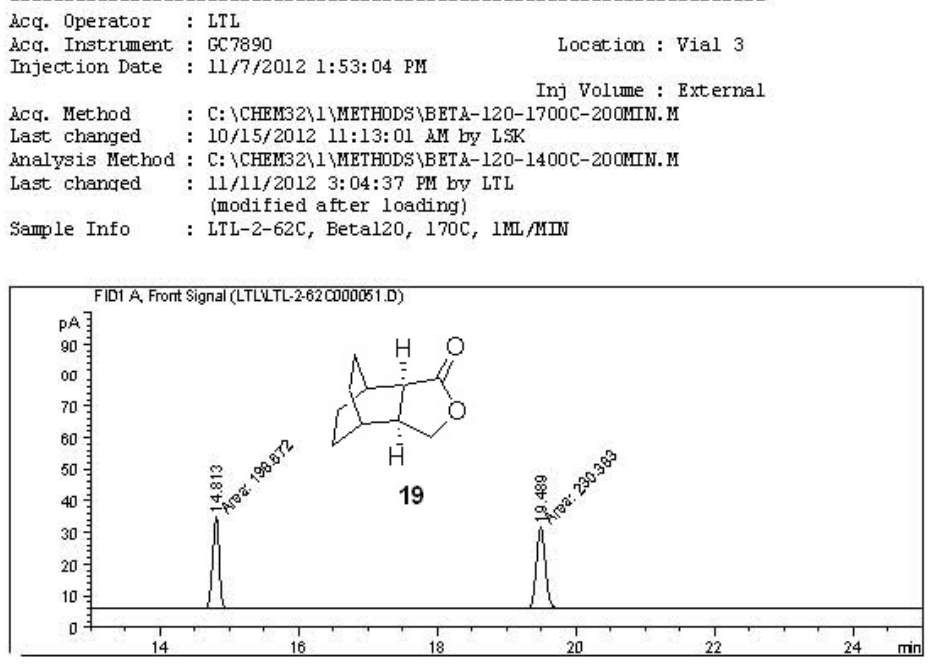

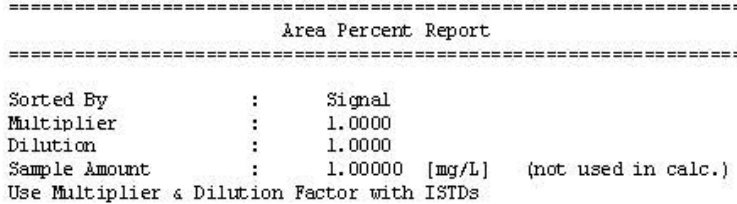

Use Phultiplier s Dilution Factor with ISTDs

Signal 1: FIDl A, Front sigmal

\begin{tabular}{|c|c|c|c|c|c|}
\hline $\begin{array}{c}\text { Peak } \\
\#\end{array}$ & $\begin{array}{l}\text { Ret Time Type } \\
\text { [min] }\end{array}$ & $\begin{array}{l}\text { Width } \\
\text { [min] }\end{array}$ & $\begin{array}{r}\text { Area } \\
{\left[\mathrm{pA}^{*} s\right]}\end{array}$ & $\begin{array}{l}\text { Height } \\
\text { [pA] }\end{array}$ & $\underset{\substack{\text { Area } \\
\mathrm{g}}}{ }$ \\
\hline 1 & $14.813 \mathrm{~mm}$ & 0.1144 & 198.67223 & 3324 & 459 \\
\hline 2 & $19.489 \mathrm{MM}$ & 0.1497 & 230.38289 & 25.64468 & 53.69541 \\
\hline
\end{tabular}

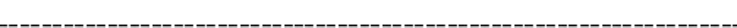

校 End of Report tהt 
Data File C: $\backslash$ CHEM $32 \backslash 1 \backslash$ DATA $\backslash L T L \backslash L T L-2-64$ C000060.D Sample Name: LTL-2-64C
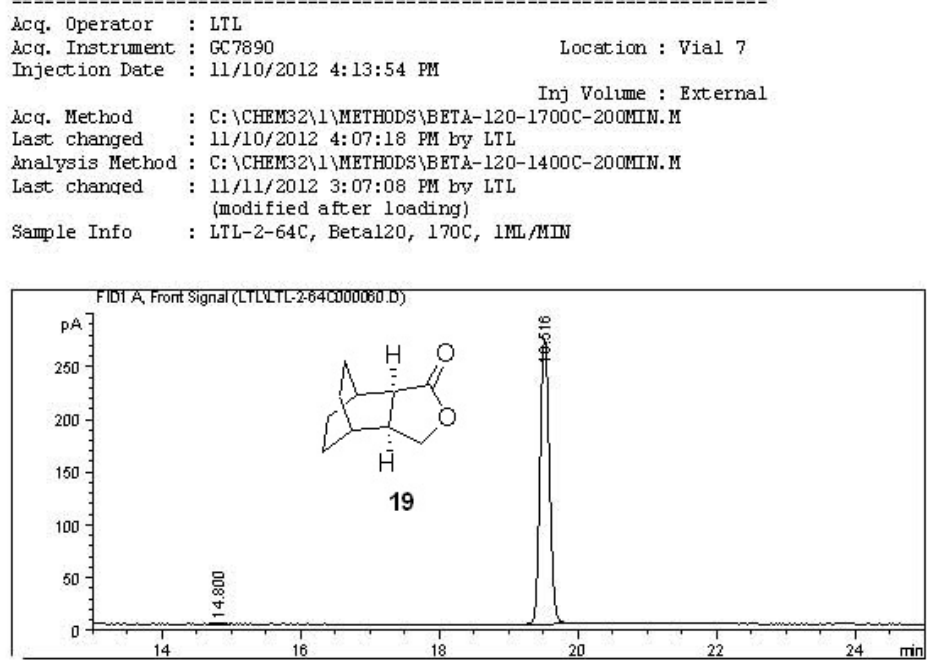

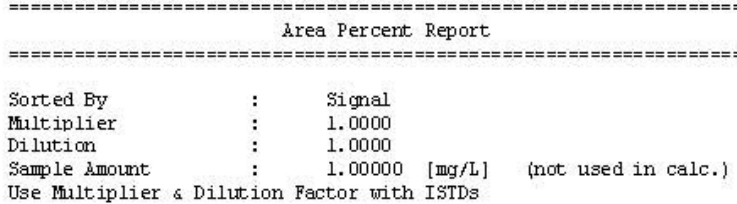

Use Pultiplier \& Dilution Factor with ISTDs

Signal 1: FIDl A, Front Sigmal

\begin{tabular}{|c|c|c|c|c|c|}
\hline $\begin{array}{c}\text { Peak } \\
\#\end{array}$ & $\begin{array}{l}\text { RetTime Type } \\
\text { [min] }\end{array}$ & $\begin{array}{l}\text { Width } \\
\text { [min] }\end{array}$ & $\begin{array}{r}\text { Area } \\
{\left[\mathrm{p}^{*} s\right]}\end{array}$ & $\begin{array}{l}\text { Height } \\
\text { [pA] }\end{array}$ & $\underset{\substack{\text { a } \\
\text { Area }}}{ }$ \\
\hline 1 & $14.800 \mathrm{BB}$ & 0.1034 & 2.72547 & 4.17 & 642 \\
\hline 2 & $19.516 \mathrm{BB}$ & 0.1471 & 2558.25391 & 270.59009 & 99.89358 \\
\hline
\end{tabular}

Totals :

$2560.97938 \quad 271.00723$

t*t End of Report 